\title{
SOME LIGHTLIKE SUBMANIFOLDS OF ALMOST COMPLEX MANIFOLDS WITH NORDEN METRIC
}

\author{
Galia Nakova \\ University of Veliko Tarnovo "St. Cyril and St. Metodius" \\ Faculty of Mathematics and Informatics, \\ T. Tarnovski 2 str. \\ 5000 Veliko Tarnovo, Bulgaria \\ E-mail:gnakova@gmail.com
}

\begin{abstract}
In this paper we study submanifolds of an almost complex manifold with Norden metric which are non-degenerate with respect to the one Norden metric and lightlike with respect to the other Norden metric on the manifold. Relations between the induced geometric objects of some of these submanifolds are given. Examples of the considered submanifolds are constructed. MSC: 53B25, 53C50, 53B50, 53C42, 53C15
\end{abstract}

Keywords: Lightlike submanifolds; Almost complex manifolds; Norden metric;

\section{Introduction}

The general theory of lightlike submanifolds has been developed in ${ }^{1}$ by $\mathrm{K}$. Duggal and A. Bejancu. The geometry of Cauchy-Riemann (CR) lightlike submanifolds of indefinite Kaehler manifolds was presented in ${ }^{1}$, too. Many new types of lightlike submanifolds of indefinite Kaehler manifolds, indefinite Sasakian and indefinite quaternion Kaehler manifolds are introduced in $^{2}$ by K. Duggal and B. Sahin. $\mathrm{In}^{1}$ and $^{2}$, different applications of lightlike geometry in the mathematical physics are given. However, lightlike submanifolds of almost complex manifolds with Norden metric (or B-metric) have not been considered yet. The study of such submanifolds is interesting because there exists a difference between the geometry of a $2 n$-dimensional indefinite almost Hermitian manifold and the geometry of a $2 n$-dimensional almost complex manifold with Norden metric. The difference arises due to the fact that in the first case, the almost complex structure $\bar{J}$ is an isometry with respect to the semi-Riemannian metric $\bar{g}$ of index $2 q(0<2 q<2 n)$ and in the second case, $\bar{J}$ is an anti-isometry with respect to the metric $\bar{g}$, which is necessarily of signature $(n, n)$. This property of the pair $(\bar{J}, \bar{g})$ 
of an almost complex manifold with Norden metric $\bar{M}$ allows to define the tensor field $\overline{\widetilde{g}}$ on $\bar{M}$ by $\overline{\widetilde{g}}(X, Y)=\bar{g}(\bar{J} X, Y)$, which is a Norden metric, too.

Let $M$ be a real $m$-dimensional submanifold of an almost complex manifold witn Norden metric $(\bar{M}, \bar{J}, \bar{g}, \overline{\widetilde{g}})$. The geometry of $M$ depends on the behaviour of the tangent bundle of $M$ with respect to the action of the almost complex structure $\bar{J}$ and the induced metric on $M$. Due to that there exist two Norden metrics $\bar{g}$ and $\overline{\widetilde{g}}$ of $\bar{M}$ we can consider two induced metrics $g$ and $\widetilde{g}$ on $M$ by $\bar{g}$ and $\overline{\widetilde{g}}$, respectively. For a submanifold $M$ of $\bar{M}$ three cases with respect to the induced metrics $g$ and $\widetilde{g}$ on $M$ are possible: $M$ is non-degenerate with respect to both $g$ and $\widetilde{g} ; M$ is degenerate with respect to both $g$ and $\widetilde{g} ; M$ is non-degenerate with respect to $g$ (resp. $\widetilde{g}$ ) and degenerate with respect to $\widetilde{g}$ (resp. $g$ ).

In this paper we consider mainly submanifolds of the third type. In Sections 1 and 2 we recall some preliminaries about lightlike submanifolds of semi-Riemannian manifolds and almost complex manifolds with Norden metric, respectively. The main results of the paper are given in Section 3 . We prove that a necessary and sufficient condition for the submanifold $(M, g)$ of $\bar{M}$ to be a CR-submanifold is $(M, \widetilde{g})$ to be a Radical transversal lightlike submanifold of $\bar{M}$. We obtain also that the submanifold $(M, g)$ of $\bar{M}$ is generic, totally real or Lagrangian if and only if $(M, \widetilde{g})$ is a coisotropic, an isotropic or a totally lightlike submanifold of $\bar{M}$, respectively. In Section 4 , in the case of totally real $(M, g)$ and isotropic $(M, \widetilde{g})$, relations between the induced geometric objects are found. The structure equations of these submanifolds of a Kaehler manifold with Norden metric are obtained. In the last section we consider known matrix Lie subgroups of $G L(2 ; \mathbb{C})$ as examples of the submanifolds introduced in Section 3.

\section{Lightlike submanifolds of semi-Riemannian manifolds}

Follow ${ }^{1}$ we give some basic notions and formulas for lightlike submanifolds of semi-Riemannian manifolds.

Let $(\bar{M}, \bar{g})$ be a real $(m+n)$-dimensional semi-Riemannian manifold $(m>1, n \geq 1)$, i.e. $\bar{g}$ is a semi-Riemannian metric of constant index $q \in\{1, \ldots, m+n-1\}$ and $M$ be a submanifold of $\bar{M}$ of codimension $n$. Denote by $g$ the induced tensor field on $M$ of $\bar{g}$ and suppose that rank $g=$ const on $M$. If rank $g=m$, i.e. $g$ is non-degenerate on the tangent bundle $T M$ of $M$, then $M$ is called a non-degenerate submanifold of $\bar{M}$. In the case $\operatorname{rank} g<m$, i.e. $g$ is degenerate on $T M$, then $M$ is called a lightlike submanifold of $\bar{M}$. We will note an orthogonal direct sum by $\perp$ and a nonorthogonal direct sum by $\oplus$. The tangent space $T_{x} M$ and the normal space 
$T_{x} M^{\perp}$ of a non-degenerate submanifold $M$ of $\bar{M}$ are non-degenerate and they are complementary orthogonal vector subspaces of $T_{x} \bar{M}$. However, if $M$ is a lightlike submanifold of $\bar{M}$, both $T_{x} M$ and $T_{x} M^{\perp}$ are degenerate orthogonal, but non-complementary subspaces of $T_{x} \bar{M}$ and there exists a subspace $\operatorname{Rad} T_{x} M=T_{x} M \cap T_{x} M^{\perp}=\operatorname{Rad} T_{x} M^{\perp}$, where

$$
\operatorname{Rad} T_{x} M=\left\{\xi_{x} \in T_{x} M: g\left(\xi_{x}, X\right)=0, \forall X \in T_{x} M\right\} .
$$

The dimension of $\operatorname{Rad} T_{x} M$ depends on $x \in M$. The submanifold $M$ of $\bar{M}$ is said to be an r-lightlike (an r-degenerate, an r-null) submanifold if the mapping

$$
\operatorname{Rad} T M: x \in M \longrightarrow \operatorname{Rad} T_{x} M,
$$

defines a smooth distribution on $M$ of rank $r>0$ (it means $\operatorname{dim}\left(\operatorname{Rad} T_{x} M\right)=r$ for all $\left.x \in M\right) . \operatorname{Rad} T M$ is called the Radical (lightlike, null) distribution on $M$.

Let $S(T M)$ be a complementary distribution of $\operatorname{Rad} T M$ in $T M$ and $S\left(T M^{\perp}\right)$ be a complementary vector bundle of $\operatorname{Rad} T M$ in $T M^{\perp}$. Both $S(T M)$ and $S\left(T M^{\perp}\right)$ are non-degenerate with respect to $\bar{g}$ and the following decompositions are valid

$$
T M=\operatorname{Rad} T M \perp S(T M), \quad T M^{\perp}=\operatorname{Rad} T M \perp S\left(T M^{\perp}\right) .
$$

The distribution $S(T M)$ and the vector bundle $S\left(T M^{\perp}\right)$ are called a screen distribution and a screen transversal vector bundle of $M$, respectively. Although $S(T M)$ is not unique, it is canonically isomorphic to the factor vector bundle $T M / \operatorname{Rad} T M$. As $S(T M)$ is a non-degenerate vector subbundle of $T \bar{M}$ we have

$$
T \bar{M}=S(T M) \perp S(T M)^{\perp},
$$

where $S(T M)^{\perp}$ is the complementary orthogonal vector bundle of $S(T M)$ in $T \bar{M}$. As $S\left(T M^{\perp}\right)$ is a vector subbundle of $S(T M)^{\perp}$ and since both are non-degenerate we have the following orthogonal direct decomposition

$$
S(T M)^{\perp}=S\left(T M^{\perp}\right) \perp S\left(T M^{\perp}\right)^{\perp} .
$$

Let $\operatorname{tr}(T M)$ and $\operatorname{lt}(T M)$ be complementary (but never orthogonal) vector bundles to $T M$ in $T \bar{M}$ and to $\operatorname{Rad} T M$ in $S\left(T M^{\perp}\right)^{\perp}$, respectively. Then we have

$$
\begin{aligned}
& \operatorname{tr}(T M)=\operatorname{ltr}(T M) \perp S\left(T M^{\perp}\right) ; \\
& T \bar{M}=T M \oplus \operatorname{tr}(T M)=S(T M) \perp S\left(T M^{\perp}\right) \perp(\operatorname{Rad} T M \oplus \operatorname{ltr}(T M)) .
\end{aligned}
$$


The vector bundle $\operatorname{ltr}(T M)$ is called a lightlike transversal vector bundle of $M$ and $\operatorname{tr}(T M)$ is called a transversal vector bundle of $M$. There exists a local quasi-orthonormal basis ${ }^{1}$ of $\bar{M}$ along $M$ :

$$
\left\{\xi_{i}, N_{i}, X_{a}, W_{\alpha}\right\}, i \in\{1, \ldots, r\}, a \in\{r+1, \ldots, m\}, \alpha \in\{r+1, \ldots, n\},
$$

where $\left\{\xi_{i}\right\}$ and $\left\{N_{i}\right\}$ are lightlike basises of $\operatorname{Rad} T M_{\mid U}$ and $\operatorname{ltr}(T M)_{\mid U}$, respectively; $\left\{X_{a}\right\}$ and $\left\{W_{\alpha}\right\}$ are basises of $S(T M)_{\mid U}$ and $S\left(T M^{\perp}\right)_{\mid U}$.

The following possible four cases with respect to the dimension $m$ and codimension $n$ of $M$ and rank $r$ of $\operatorname{Rad} T M$ are studied:

(1) $r$-lightlike, if $0<r<\min (m, n)$;

(2) coisotropic, if $1 \leq r=n<m, \quad S\left(T M^{\perp}\right)=\{0\}$;

(3) isotropic, if $1<r=m<n, \quad S(T M)=\{0\}$;

(4) totally lightlike, if $1<r=m=n, \quad S(T M)=\{0\}=S\left(T M^{\perp}\right)$.

\section{Almost complex manifolds with Norden metric}

Let $(\bar{M}, \bar{J}, \bar{g})$ be a $2 n$-dimensional almost complex manifold with Norden metric $^{3}$, i.e. $\bar{J}$ is an almost complex structure and $\bar{g}$ is a metric on $\bar{M}$ such that:

$$
\bar{J}^{2} X=-X, \quad \bar{g}(\bar{J} X, \bar{J} Y)=-\bar{g}(X, Y)
$$

for arbitrary differentiable vector fields $X, Y$ on $\bar{M}$.

The tensor field $\overline{\widetilde{g}}$ of type $(0,2)$ on $\bar{M}$ defined by

$$
\overline{\widetilde{g}}(X, Y)=\bar{g}(\bar{J} X, Y)
$$

is a Norden metric on $\bar{M}$, too. Both metrics $\bar{g}$ and $\overline{\widetilde{g}}$ are necessarily of signature $(n, n)$. The metric $\overline{\widetilde{g}}$ is said to be an associated metric of $\bar{M}$. The Levi-Civita connection of $\bar{g}$ is denoted by $\bar{\nabla}$. The tensor field $F$ of type $(0,3)$ on $\bar{M}$ is defined by $F(X, Y, Z)=\bar{g}\left(\left(\bar{\nabla}_{X} \bar{J}\right) Y, Z\right)$. Let $\overline{\widetilde{\nabla}}$ be the Levi-Civita connection of $\overline{\widetilde{g}}$. Then

$$
\Phi(X, Y)=\bar{\nabla}_{X} Y-\bar{\nabla}_{X} Y
$$

is a tensor field of type $(1,2)$ on $\bar{M}$. Since $\bar{\nabla}$ and $\bar{\nabla}$ are torsion free we have $\Phi(X, Y)=\Phi(Y, X)$. A classification of the almost complex manifolds with Norden metric with respect to the tensor $F$ is given in $^{3}$ and eight classes 
are obtained. $\mathrm{In}^{4}$ these classes are characterized by conditions for the tensor $\Phi$, too.

Analogously, as for an almost Hermitian manifold, for an almost complex manifold with Norden metric $(\bar{M}, \bar{J}, \bar{g}, \overline{\widetilde{g}})$ the following important classes of non-degenerate submanifolds of $\bar{M}$ with respect to the induced metric $g$ (resp. $\widetilde{g}$ ) on the submanifold can be considered:

(a) $M$ is called a holomorphic (or an invariant) submanifold of $\bar{M}$ if $\bar{J}\left(T_{x} M\right)=T_{x} M, \forall x \in M$. The dimension $m$ of an invariant submanifold $M$ of $\bar{M}$ is necessarily even;

(b) $M$ is called a totally real (or an anti-invariant) submanifold of $\bar{M}$ if $\bar{J}\left(T_{x} M\right) \subseteq T_{x} M^{\perp}, \forall x \in M$. In this case $\operatorname{dim} M=m \leq n ;$

(c) A totally real submanifold $M$ is called Lagrangian if $\operatorname{dim} M=m=n$;

(d) $M$ is called a $C R$-submanifold of $\bar{M}$ if $M$ is endowed with two complementary orthogonal distributions $D$ and $D^{\perp}$ satisfying the conditions: $\bar{J}\left(D_{x}\right)=D_{x}$ and $\bar{J}\left(D_{x}^{\perp}\right) \subset T_{x} M^{\perp}, \quad \forall x \in M ;$

(e) The CR-submanifold $M$ of $\bar{M}$ is called a generic submanifold if $\operatorname{dim} D^{\perp}=\operatorname{codim} M=2 n-m$.

The CR-submanifold $M$ of $\bar{M}$ is called non-trivial (proper) if $\operatorname{dim} D>0$ and $\operatorname{dim} D^{\perp}>0$. The holomorphic and the totally real submanifolds are particular cases of the class of CR-submanifolds. Holomorphic submanifolds of almost complex manifolds with Norden metric were studied by K. Gribachev. In $^{5},{ }^{6}$ and $^{7}$ hypersurfaces of Kaehler manifolds with Norden metric were considered.

\section{Submanifolds of an almost complex manifold with Norden metric which are non-degenerate with respect to the one Norden metric and lightlike with respect to the other Norden metric}

Let $(\bar{M}, \bar{J}, \bar{g}, \overline{\widetilde{g}})$ be a $2 n$-dimensional almost complex manifold with Norden metric and $M$ be an $m$-dimensional submanifold immersed by $\varphi$ in $\bar{M}$. For simplicity we identify for each $x \in M$ the tangent space $T_{x} M$ with $\varphi *\left(T_{x} M\right) \subset T_{\varphi(x)} \bar{M}$. Let $g$ and $\widetilde{g}$ be two metrics of $M$. We assume that the immersion $\varphi$ is an isometry with respect to both metrics $\bar{g}$ and $\overline{\widetilde{g}}$ on $\bar{M}$ and we identify the metrics $g$ and $\widetilde{g}$ on $M$ with the induced metrics on the subspace $\varphi *\left(T_{x} M\right)$ of $\bar{g}$ and $\overline{\widetilde{g}}$, respectively. Hence, for any $x \in M$ we have

$$
g\left(X_{x}, Y_{x}\right)=\bar{g}\left(X_{x}, Y_{x}\right), \quad \widetilde{g}\left(X_{x}, Y_{x}\right)=\overline{\widetilde{g}}\left(X_{x}, Y_{x}\right), \quad \forall X_{x}, Y_{x} \in T_{x} M
$$


We note that $T M=\bigcup_{x \in M} T_{x} M$ is the tangent bundle of both submanifolds $(M, g)$ and $(M, \widetilde{g})$ of $\bar{M}$. We will denote: the normal bundle of $(M, g)$ and $(M, \widetilde{g})$ by $T M^{\perp}$ and $T M^{\widetilde{\perp}}$, respectively; an orthogonal direct sum with respect to $\bar{g}$ (resp. $\overline{\widetilde{g}}$ ) by $\perp$ (resp. $\widetilde{\perp}$ ) and a non-orthogonal direct sum by $\oplus$ (resp. $\widetilde{\oplus})$.

In this section we give an answer to the question what type is the submanifold $(M, \widetilde{g})$ of $\bar{M}$ if $(M, g)$ belongs to one of the basic classes of nondegenerate submanifolds.

Lemma 3.1. Let $(V, J, \bar{g}, \overline{\widetilde{g}})$ be a $2 n$-dimensional almost complex vector space with Norden metric and $W$ be a $2 p$-dimensional holomorphic subspace of $V$. Then for the induced metrics $g$ and $\widetilde{g}$ on $W$ of $\bar{g}$ and $\overline{\widetilde{g}}$ respectively, we have

1) $g$ is non-degenerate iff $\widetilde{g}$ is non-degenerate;

2) $g$ is degenerate iff $\widetilde{g}$ is degenerate.

Proof. 1) Let $g$ be non-degenerate. We assume that $\widetilde{g}$ is degenerate. Then there exists $\xi \in W, \xi \neq 0$ such that $\widetilde{g}(\xi, X)=0, \forall X \in W$. As $W$ is a holomorphic subspace of $V$ and $\operatorname{Ker} J=\{0\}$ it follows that there exists $J \xi \in W, J \xi \neq 0$ such that for $\forall X \in W$ we have $g(J \xi, X)=\bar{g}(J \xi, X)=$ $\overline{\widetilde{g}}(\xi, X)=\widetilde{g}(\xi, X)=0$. So, we obtain that $g$ is degenerate, which is a contradiction. Analogously, we can check that if $\widetilde{g}$ is non-degenerate, then $g$ is non-degenerate, too. The truth of 2) follows from 1).

Theorem 3.1. Let $(\bar{M}, \bar{J}, \bar{g}, \overline{\widetilde{g}})$ be a $2 n$-dimensional almost complex manifold with Norden metric and $M$ be a $2 p$-dimensional submanifold of $\bar{M}$. The submanifold $(M, g)$ is holomorphic iff the submanifold $(M, \widetilde{g})$ is holomorphic.

Proof. The proof of the theorem follows from assertion 1) of Lemma 3.1 by replacing the space $V$ and the subspace $W$ by the tangent bundle $T \bar{M}$ of $\bar{M}$ and the tangent bundle $T M$ of the submanifolds $(M, g)$ and $(M, \widetilde{g})$, respectively.

Radical transversal lightlike submanifolds of indefinite Kaehler manifolds are introduced in ${ }^{8}$ by Sahin. Further, we show that such submanifolds naturally arise on an almost complex manifold with Norden metric $\bar{M}$ and they are related with CR-submanifolds of $\bar{M}$. First, analogously as in $^{8}$ we give the following 
Definition 3.1. Let $\left(M, g, S(T M), S\left(T M^{\perp}\right)\right)$ be a lightlike submanifold of an almost complex manifold with Norden metric $(\bar{M}, \bar{J}, \bar{g}, \overline{\widetilde{g}})$. We say that $M$ is a Radical transversal lightlike submanifold of $\bar{M}$ if the following conditions are satisfied:

$$
\begin{gathered}
\bar{J}(\operatorname{Rad} T M)=\operatorname{ltr}(T M), \\
\bar{J}(S(T M))=S(T M) .
\end{gathered}
$$

Moreover, we say that a Radical transversal lightlike submanifold $M$ is proper if $S(T M) \neq 0$.

It is important to note:

(1) Taking into account that for an isotropic and a totally lightlike submanifold $S(T M)=0$ and Definition 3.1, it is clear that there exists no proper Radical transversal isotropic and totally lightlike submanifold of $\bar{M}$.

(2) Contrary to the case when $M$ is a Radical transversal lightlike submanifold of an indefinite Kaehler manifold ${ }^{8}$, for an almost complex manifold with Norden metric $(\bar{M}, \bar{J}, \bar{g}, \bar{g}), M$ can be an 1-lightlike Radical transversal lightlike submanifold of $\bar{M}$. Indeed, let us suppose that $(M, g)$ is an 1-lightlike Radical transversal lightlike submanifold of $(\bar{M}, \bar{J}, \bar{g}, \overline{\widetilde{g}})$. Then there exist basises $\{\xi\}$ and $\{N\}$ of $\operatorname{Rad} T M$ and $\operatorname{ltr}(T M)$ respectively, such that $\bar{g}(\xi, N)=1$. On the other hand, (6) implies that $\bar{J} \xi=\alpha N \in \Gamma(\operatorname{ltr}(T M)), \alpha \in F(\operatorname{ltr}(T M))$. Thus, for the function $\alpha$ we obtain $\alpha=\bar{g}(\xi, \bar{J} \xi)$, which is not zero.

Proposition 3.1. Let $(M, g)$ be a Radical transversal lightlike submanifold of an almost complex manifold with Norden metric $(\bar{M}, \bar{J}, \bar{g}, \overline{\widetilde{g}})$. Then the distribution $S\left(T M^{\perp}\right)$ is holomorphic with respect to $\bar{J}$.

Proof. As the tangent bundle $T M$ and the transversal vector bundle $\operatorname{tr}(T M)$ of $(M, g)$ are complementary (but not orthogonal with respect to $\bar{g})$ vector subbundles of $T \bar{M}$, for any $X \in \Gamma(T M)$ and any $V \in \Gamma(\operatorname{tr}(T M))$, we have

$$
\bar{J} X=T X+F X ; \quad \bar{J} V=t V+f V,
$$

where $T X, t V$ belong to $\Gamma(T M)$ and $F X, f V$ belong to $\Gamma(\operatorname{tr}(T M))$. Then $T$ and $f$ are endomorphisms on $T M$ and $\operatorname{tr}(T M)$, respectively; $F$ and $t$ are transversal bundle-valued 1-form on $T M$ and tangent bundle-valued 1-form 
on $\operatorname{tr}(T M)$, respectively. According to the decompositions (1), (2), we can write any $X \in \Gamma(T M)$ and any $V \in \Gamma(\operatorname{tr}(T M))$ in the following manner

$$
X=P X+Q X ; \quad V=L V+S V,
$$

where $P$ and $Q$ are the projection morphisms of $T M$ on $S(T M)$ and $\operatorname{Rad} T M$, respectively; $L$ and $S$ are the projection morphisms of $\operatorname{tr}(T M)$ on $\operatorname{ltr}(T M)$ and $S\left(T M^{\perp}\right)$, repectively. So, for any $W \in \Gamma S\left(T M^{\perp}\right)$ we have

$$
\bar{J} W=t W+f W=P t W+Q t W+L f W+S f W .
$$

Now, if $X \in \Gamma S(T M), \xi \in \Gamma(\operatorname{Rad} T M), N \in \Gamma(\operatorname{ltr}(T M))$, using (2), (6), (7), (10) and $\bar{J}(\operatorname{ltr}(T M))=\operatorname{Rad} T M$, we compute

$$
\begin{aligned}
& 0=\bar{g}(W, \bar{J} X)=\bar{g}(\bar{J} W, X)=\bar{g}(P t W, X), \\
& 0=\bar{g}(W, \bar{J} \xi)=\bar{g}(\bar{J} W, \xi)=\bar{g}(L f W, \xi), \\
& 0=\bar{g}(W, \bar{J} N)=\bar{g}(\bar{J} W, N)=\bar{g}(Q t W, N) .
\end{aligned}
$$

Since $\bar{g}$ is non-degenerate on $S(T M)$ and $\operatorname{Rad} T M \oplus \operatorname{ltr}(T M)$, from (11) it follows that $P t W=L f W=Q t W=0$. Then (10) becomes $\bar{J} W=S f W$, which means that $S\left(T M^{\perp}\right)$ is holomorphic.

Theorem 3.2. Let $(\bar{M}, \bar{J}, \bar{g}, \overline{\widetilde{g}})$ be a $2 n$-dimensional almost complex manifold with Norden metric and $M$ be an m-dimensional submanifold of $\bar{M}$. The submanifold $(M, g)$ is a CR-submanifold with an $r$-dimensional totally real distribution $D^{\perp}$ iff $(M, \widetilde{g})$ is an r-lightlike Radical transversal lightlike submanifold.

Proof. As far as we know, CR-submanifolds of almost complex manifolds with Norden metric are not studied. Therefore, first we give some preliminaries about them.

Let $(M, g)$ be a CR-submanifold of $\bar{M}$ and assume that it is not generic. Hence, $(M, g)$ is endowed with two complementary orthogonal with respect to $\bar{g}$ distributions $D(\operatorname{dim} D=2 p)$ and $D^{\perp}\left(\operatorname{dim} D^{\perp}=r: 1 \leq r<\right.$ $\min (m, 2 n-m))$ such that $\bar{J} D=D, \bar{J} D^{\perp} \subset T M^{\perp}$. Following ${ }^{9}$, for any $X \in \Gamma(T M)$ we have

$$
\bar{J} X=T X+F X
$$

where $T X$ is the tangential part of $\bar{J} X$ and $F X$ is the normal part of $\bar{J} X$. Then $T$ is an endomorphism on the tangent bundle $T M$ and $F$ is a normal bundle-valued 1-form on $T M$. Similarly, for any $V \in \Gamma\left(T M^{\perp}\right)$ we have

$$
\bar{J} V=t V+f V,
$$


where $t V$ is the tangential part of $\bar{J} V$ and $f V$ is the normal part of $\bar{J} V$. Then $f$ is an endomorphism on the normal bundle $T M^{\perp}$ and $t$ is a tangent bundle-valued 1-form on $T M^{\perp}$. If we denote by $P$ and $Q$ the projection morphisms of $T M$ on $D$ and $D^{\perp}$ respectively, for any $X \in \Gamma(T M)$ we can write

$$
X=P X+Q X,
$$

where $P X \in \Gamma(D)$ and $Q X \in \Gamma\left(D^{\perp}\right)$. Using (12) and (13), for $Y \in \Gamma(T M)$ and $U \in \Gamma\left(T M^{\perp}\right)$ we compute $\bar{g}(\bar{J} X, Y)=\bar{g}(T X, Y)$ and $\bar{g}(\bar{J} V, U)=$ $\bar{g}(f V, U)$. Since the almost complex structure $\bar{J}$ is an anti-isometry with respect to the Norden metrics $\bar{g}$ and $\overline{\widetilde{g}}$, we obtain that $T$ and $f$ are self-adjoint operators on $T M$ and $T M^{\perp}$ with respect to both metrics. We note that $T$ and $f$ are skew self-adjoint ${ }^{9}$ when $\bar{M}$ is an almost Hermitian manifold. We also find $\bar{g}(F X, V)=\bar{g}(X, t V)$. Moreover, applying $\bar{J}$ to the equality (14) we obtain

$$
\bar{J} X=\bar{J} P X+\bar{J} Q X
$$

where $\bar{J} P X \in \Gamma(D)$ and $\bar{J} Q X \in \Gamma\left(\bar{J} D^{\perp}\right)$. From (12) and (15) it follows that $T=\bar{J} P$ and $F=\bar{J} Q$. Due to the fact that $D^{\perp}$ is non-degenerate with respect to $\bar{g}$, there exists an orthonormal basis $\left\{\xi_{1}, \ldots, \xi_{r}\right\}$ of $D^{\perp}$, i.e.

$$
g\left(\xi_{i}, \xi_{i}\right)=\epsilon_{i}, \epsilon_{i}= \pm 1 ; \quad g\left(\xi_{i}, \xi_{j}\right)=0, i \neq j ; \quad i, j=1,2, \ldots, r .
$$

Since $\operatorname{Ker} \bar{J}=\{0\},\left\{\bar{J} \xi_{1}, \ldots, \bar{J} \xi_{r}\right\}$ is a basis of $\bar{J} D^{\perp}$. Moreover, it is an orthonormal basis such that

$$
\bar{g}\left(\bar{J} \xi_{i}, \bar{J} \xi_{i}\right)=-\epsilon_{i} ; \quad \bar{g}\left(\bar{J} \xi_{i}, \bar{J} \xi_{j}\right)=0, i \neq j ; \quad i, j=1,2, \ldots, r .
$$

Consequently, $\bar{J} D^{\perp}$ is an $r$-dimensional non-degenerate subbundle of $T M^{\perp}$ with respect to $\bar{g}$ and we put

$$
T M^{\perp}=\bar{J} D^{\perp} \perp\left(\bar{J} D^{\perp}\right)^{\perp},
$$

where $\left(\bar{J} D^{\perp}\right)^{\perp}$ is the complementary orthogonal vector subbundle of $\bar{J} D^{\perp}$ in $T M^{\perp}$. We denote by $P_{1}$ and $P_{2}$ the projection morphisms of $T M^{\perp}$ on $\bar{J} D^{\perp}$ and $\left(\bar{J} D^{\perp}\right)^{\perp}$, respectively. Then for any $V \in \Gamma\left(T M^{\perp}\right)$ we put

$$
V=P_{1} V+P_{2} V
$$

where $P_{1} V \in \Gamma\left(\bar{J} D^{\perp}\right)$ and $P_{2} V \in \Gamma\left(\left(\bar{J} D^{\perp}\right)^{\perp}\right)$. Now, we will show that the subbundle $\left(\bar{J} D^{\perp}\right)^{\perp}$ is holomorphic with respect to $\bar{J}$. Take $W \in$ $\Gamma\left(\left(\bar{J} D^{\perp}\right)^{\perp}\right), X \in \Gamma(T M)$ and according to $(15)$ we compute $\bar{g}(\bar{J} W, X)=$ $\bar{g}(W, \bar{J} X)=0$, which means that $\bar{J} W \in \Gamma\left(T M^{\perp}\right)$. On the other hand, for each $N \in \Gamma\left(\bar{J} D^{\perp}\right)$ we have $\bar{J} N \in \Gamma\left(D^{\perp}\right)$, which implies $\bar{g}(\bar{J} W, N)=$ 
$\bar{g}(W, \bar{J} N)=0$, i.e. $\bar{J} W \in \Gamma\left(\left(\bar{J} D^{\perp}\right)^{\perp}\right)$. We continue by proving that the normal bundle $T M^{\mathcal{\perp}}$ of the submanifold $(M, \widetilde{g})$ coincides with the subbundle $D^{\perp} \perp\left(\bar{J} D^{\perp}\right)^{\perp}$ of $T \bar{M}$. First, let us suppose that $\bar{Y} \in \Gamma\left(D^{\perp} \perp\left(\bar{J} D^{\perp}\right)^{\perp}\right)$. Then for $\forall X \in \Gamma(T M)$ using (15) we have $\overline{\widetilde{g}}(X, \bar{Y})=\bar{g}(\bar{J} X, \bar{Y})=0$. Hence, $\bar{Y} \in \Gamma\left(T M^{\widetilde{\perp}}\right)$, i.e. the following relation holds

$$
D^{\perp} \perp\left(\bar{J} D^{\perp}\right)^{\perp} \subseteq T M^{\tilde{\perp}} .
$$

Now, let $\bar{Y} \in \Gamma\left(T M^{\widetilde{\perp}}\right)$. Then $\bar{Y} \in \Gamma(T \bar{M})$ and $\overline{\widetilde{g}}(X, \bar{Y})=0$ for $\forall X \in \Gamma(T M)$. The last is equivalent to $\bar{g}(X, \overline{J Y})=0$ which shows that $\overline{J Y} \in \Gamma\left(T M^{\perp}\right)$. Since $\left(\bar{J} D^{\perp}\right)^{\perp}$ is holomorphic with respect to $\bar{J}$, applying $\bar{J}$ to $(18)$ we obtain $\bar{J}\left(T M^{\perp}\right)=D^{\perp} \perp\left(\bar{J} D^{\perp}\right)^{\perp}$. So, we conclude that $\bar{Y} \in \Gamma\left(D^{\perp} \perp\left(\bar{J} D^{\perp}\right)^{\perp}\right)$ and we have

$$
T M^{\widetilde{\perp}} \subseteq D^{\perp} \perp\left(\bar{J} D^{\perp}\right)^{\perp} .
$$

From (20) and (21) it follows that $T M^{\widetilde{\perp}}=D^{\perp} \perp\left(\bar{J} D^{\perp}\right)^{\perp}$. Further, for $X \in$ $\Gamma(D), \xi \in \Gamma\left(D^{\perp}\right)$ and $W \in \Gamma\left(\left(\bar{J} D^{\perp}\right)^{\perp}\right)$, we get $\overline{\widetilde{g}}(\xi, X)=\bar{g}(\bar{J} \xi, X)=0$, $\overline{\widetilde{g}}(\xi, W)=\bar{g}(\bar{J} \xi, W)=0$. The last two equalities show that $D^{\perp}$ is orthogonal to $D$ and $\left(\bar{J} D^{\perp}\right)^{\perp}$ with respect to $\overline{\widetilde{g}}$. Then the following decompositions are valid

$$
\begin{gathered}
T M=D \widetilde{\perp} D^{\perp}, \\
T M^{\widetilde{\perp}}=D^{\perp} \widetilde{\perp}\left(\bar{J} D^{\perp}\right)^{\perp} .
\end{gathered}
$$

From $(22)$ and $(23)$ it is clear that the smooth distribution $D^{\perp}$ on $(M, \widetilde{g})$ of rank $r>0$ is an intersection of the tangent bundle $T M$ and the normal bundle $T M^{\widetilde{\perp}}$ of $(M, \widetilde{g})$. Hence, $(M, \widetilde{g})$ is an $r$-lightlike submanifold of $\bar{M}$ with Radical distribution $\operatorname{Rad} T M$ which coincides with $D^{\perp}$. According to (22) and (23), the distribution $D$ and the vector bundle $\left(\bar{J} D^{\perp}\right)^{\perp}$ are a screen distribution $S(T M)$ and a screen transversal vector bundle $S\left(T M^{\widetilde{\perp}}\right)$ of $(M, \widetilde{g})$, respectively. The basis $\left\{\xi_{1}, \ldots, \xi_{r}\right\}$ of $D^{\perp}$ satisfying (16) is a basis of $\operatorname{Rad} T M$ and consequently with respect to $\widetilde{g}$ we have $\widetilde{g}\left(\xi_{i}, \xi_{j}\right)=0$ for any $i, j=1, \ldots, r$. We put $N_{i}=-\epsilon_{i} \bar{J} \xi_{i}, \quad i=1, \ldots, r$. Then $\left\{N_{1}, \ldots, N_{r}\right\}$ is a basis of $\bar{J} D^{\perp}$ such that

$$
\begin{gathered}
\overline{\widetilde{g}}\left(N_{i}, N_{j}\right)=-\epsilon_{i} \epsilon_{j} \bar{g}\left(\bar{J} \xi_{i}, \xi_{j}\right)=0, \quad i, j=1, \ldots, r, \\
\overline{\widetilde{g}}\left(N_{i}, \xi_{i}\right)=\epsilon_{i} \bar{g}\left(\xi_{i}, \xi_{i}\right)=1 ; \overline{\widetilde{g}}\left(N_{i}, \xi_{j}\right)=\epsilon_{i} \bar{g}\left(\xi_{i}, \xi_{j}\right)=0 ; i, j=1, \ldots, r .
\end{gathered}
$$

From (24) it follows that $\bar{J} D^{\perp}$ is a lightlike vector bundle with respect to $\overline{\widetilde{g}}$. The equalities $(25)$ show that $\bar{J} D^{\perp}$ is not orthogonal to $\operatorname{Rad} T M$ with 
respect to $\overline{\widetilde{g}}$. It is easy also to check that $\bar{J} D^{\perp}$ is orthogonal to $S(T M)$ and $S\left(T M^{\widetilde{\perp}}\right)$ with respect to $\overline{\widetilde{g}}$. So, the tangent bundle $T \bar{M}$ of $\bar{M}$ has the following decomposition

$$
T \bar{M}=S(T M) \widetilde{\perp} S\left(T M^{\widetilde{\perp}}\right) \widetilde{\perp}\left(\operatorname{Rad} T M \widetilde{\oplus} \bar{J} D^{\perp}\right) .
$$

The equality (26) implies that $S\left(T M^{\widetilde{\perp}}\right) \widetilde{\perp}\left(\operatorname{Rad} T M \widetilde{\oplus} \bar{J} D^{\perp}\right)$ is the complementary orthogonal vector bundle $S(T M)^{\widetilde{\perp}}$ to $S(T M)$ in $T \bar{M}$ with respect to $\overline{\widetilde{g}}$. Moreover, $\operatorname{Rad} T M \widetilde{\oplus} \bar{J} D_{\widetilde{\perp}}^{\perp}$ is the complementary orthogonal vector bundle to $S\left(T M^{\widetilde{\perp}}\right)$ in $S(T M)^{\widetilde{\perp}}$ with respect to $\overline{\widetilde{g}}$, i.e.

$$
S\left(T M^{\widetilde{\perp}}\right)^{\widetilde{\perp}}=\operatorname{Rad} T M \widetilde{\oplus} \bar{J} D^{\perp} .
$$

Finally, taking into account $(27)$ we conclude that the vector bundle $\bar{J} D^{\perp}$ is the complementary (but not orthogonal with respect to $\overline{\widetilde{g}}$ ) vector bundle to $\operatorname{Rad} T M$ in $S\left(T M^{\widetilde{\perp}}\right)^{\widetilde{\perp}}$, i.e. $\bar{J} D^{\perp}$ is a lightlike transversal vector bundle of $(M, \widetilde{g})$ with respect to the pair $\left(S(T M)=D, S\left(T M^{\widetilde{\perp}}\right)=\left(\bar{J} D^{\perp}\right)^{\perp}\right)$. Hence, $(M, \widetilde{g})$ is an $r$-lightlike Radical transversal lightlike submanifold.

Conversely, let $(M, \widetilde{g})$ be an $r$-lightlike Radical transversal lightlike submanifold of $\bar{M}$. Take $X \in \Gamma(S(T M)), \xi \in \Gamma(\operatorname{Rad} T M), N \in \Gamma(\operatorname{ltr}(T M))$, $W \in \Gamma\left(S\left(T M^{\widetilde{\perp}}\right)\right)$ and using $(2),(6),(7)$ and Proposition 3.1, we get

$$
\begin{array}{ll}
\bar{g}(X, \xi)=-\overline{\widetilde{g}}(\bar{J} X, \xi)=0, & \bar{g}(X, N)=-\overline{\widetilde{g}}(\bar{J} X, N)=0, \\
\bar{g}(\xi, N)=-\overline{\widetilde{g}}(\bar{J} \xi, N)=0, & \bar{g}(W, X)=-\overline{\widetilde{g}}(\bar{J} W, X)=0, \\
\bar{g}(W, \xi)=-\overline{\widetilde{g}}(\bar{J} W, \xi)=0, & \bar{g}(W, N)=-\overline{\widetilde{g}}(\bar{J} W, N)=0 .
\end{array}
$$

The equalities (28) show that the vector bundles $S(T M), \operatorname{Rad} T M, \operatorname{ltr}(T M)$ and $S\left(T M^{\widetilde{\perp}}\right)$ are mutually orthogonal with respect to $\bar{g}$. This fact and (2) imply the following decompositions

$$
\begin{aligned}
& T M=S(T M) \perp \operatorname{Rad} T M, \quad \operatorname{tr}(T M)=\operatorname{ltr}(T M) \perp S\left(T M^{\widetilde{\perp}}\right), \\
& T \bar{M}=T M \perp \operatorname{tr}(T M) .
\end{aligned}
$$

From the last equality of (29) it follows that the normal bundle $T M^{\perp}$ of the submanifold $(M, g)$ coincides with the transversal vector bundle $\operatorname{tr}(T M)$ of $(M, \widetilde{g})$. Hence, both $T M$ and $T M^{\perp}$ are non-degenerate with respect to $\bar{g}$. The distribution $S(T M)$ is holomorphic and non-degenerate with respect to $\widetilde{g}$. According to Lemma 3.1, $S(T M)$ is non-degenerate with respect to $g$, too. If we assume that the distribution $\operatorname{Rad} T M$ is degenerate with respect to $g$, then the first equality of (29) implies that $T M$ is degenerate, which is a contradiction. Consequently, $(M, g)$ is endowed with two complementary orthogonal with respect to $g$ distributions $D^{\perp}=\operatorname{Rad} T M$ and $D=S(T M)$ satisfying (6) and (7), respectively. Since $T M^{\perp}=\operatorname{tr}(T M)$ and by using (29) 
we have $T M^{\perp}=\bar{J} T M \perp S\left(T M^{\widetilde{\perp}}\right)$. Hence, the distribution $D^{\perp}$ is totally real and moreover $S\left(T M^{\widetilde{\perp}}\right)$ is the complementary orthogonal with respect to $\bar{g}$ vector bundle of $\bar{J} T M$ in $T M^{\perp}$. So, we establish that $(M, g)$ is a CR-submanifold of $\bar{M}$.

Taking into account the definition of a generic submanifold and a coisotropic one, as an immediate consequence from Theorem 3.2, we obtain

Corollary 3.1. Let $(\bar{M}, \bar{J}, \bar{g}, \overline{\widetilde{g}})$ be a $2 n$-dimensional almost complex manifold with Norden metric and $M$ be an m-dimensional submanifold of $\bar{M}$. The submanifold $(M, g)$ is generic iff $(M, \widetilde{g})$ is a coisotropic Radical transversal lightlike submanifold.

Also we state

Theorem 3.3. Let $(\bar{M}, \bar{J}, \bar{g}, \overline{\widetilde{g}})$ be a $2 n$-dimensional almost complex manifold with Norden metric and $M$ be an $m$-dimensional submanifold of $\bar{M}$. Then

(1) The submanifold $(M, g)(1<m<n)$ is totally real iff $(M, \widetilde{g})$ is an isotropic submanifold such that $\bar{J}(T M)=\operatorname{lt}(T M)$.

(2) The submanifold $(M, g)$ is Lagrangian iff $(M, \widetilde{g})$ is a totally lightlike submanifold such that $\bar{J}(T M)=\operatorname{ltr}(T M)$.

Proof. An $m$-dimensional $(1<m<n)$ submanifold $(M, g)$ of $\bar{M}$ is totally real if it is a CR-submanifold with a holomorphic distribution $D=\{0\}$, i.e. $T M=D^{\perp}$. As $1<m<n, \bar{J} T M \subset T M^{\perp}$. On the other hand, for an isotropic submanifold $S(T M)=\{0\}$. By replacing $D$ and $S(T M)$ by zero in the proof of Theorem 3.2, we establish the truth of assertion (1). Hence, we have the following direct decompositions of the tangent bundle $T \bar{M}$ of $\bar{M}$

$$
T \bar{M}=T M \perp \bar{J} T M \perp(\bar{J} T M)^{\perp}, T \bar{M}=(T M \widetilde{\oplus} \operatorname{ltr}(T M)) \widetilde{\perp} S\left(T M^{\widetilde{\perp}}\right),
$$

where $\bar{J} T M=\operatorname{ltr}(T M)$ and $(\bar{J} T M)^{\perp}=S\left(T M^{\widetilde{\perp}}\right)$.

A Lagrangian submanifold is a particular case of a CR-submanifold, too. Now, $D=\{0\}$ and $\bar{J} D^{\perp}=\bar{J} T M=T M^{\perp}$. Hence, $(\bar{J} T M)^{\perp}=\{0\}$. So, following the proof of Theorem 3.2 and taking into account that for a totally lightlike submanifold $S(T M)=S\left(T M^{\widetilde{\perp}}\right)=\{0\}$, we verify that assertion (2) is true. 
Remark 3.1. From (4) we have $\bar{g}(X, Y)=-\overline{\widetilde{g}}(\bar{J} X, Y)$. Therefore we can interchange the metrics $\bar{g}$ (resp. $g$ ) and $\overline{\widetilde{g}}$ (resp. $\widetilde{g}$ ) in Theorem 3.2, Corollary 3.1 and Theorem 3.3.

\section{Relations between the induced geometric objects on a totally real and an isotropic submanifold of almost complex manifold with Norden metric}

Further, our purpose is to establish relations between the induced geometric objects on the submanifolds introduced in the previous section. As a first step in this direction, we start with the study of the submanifolds from assertion (1) of Theorem 3.3. In this section we consider an $m$-dimensional $(1<m<n)$ totally real submanifold $(M, g)$ of a $2 n$-dimensional almost complex manifold with Norden metric $(\bar{M}, \bar{J}, \bar{g}, \overline{\widetilde{g}})$. Then from Theorem 3.3 it follows that $(M, \widetilde{g})$ is an isotropic submanifold of $\bar{M}$ such that $\bar{J}(T M)=$ $\operatorname{ltr}(T M)$. From now on, we will denote: $\bar{X}, \bar{Y}, \bar{Z}, \bar{U} \in \Gamma(T \bar{M}) ; X, Y, Z, U \in$ $\Gamma(T M) ; V, V^{\prime} \in \Gamma\left(T M^{\perp}\right)=\Gamma(\operatorname{tr}(T M)) ; N, N^{\prime} \in \Gamma(\bar{J} T M)=\Gamma(\operatorname{ltr}(T M))$ and $W, W^{\prime} \in \Gamma\left((\bar{J} T M)^{\perp}\right)=\Gamma\left(S\left(T M^{\widetilde{\perp}}\right)\right)$.

Let $\overline{\widetilde{\nabla}}$ be the Levi-Civita connection of the metric $\overline{\tilde{g}}$ on $\bar{M}$. According to ${ }^{1}$, the formulas of Gauss and Weingarten for an $r$-lightlike submanifold $(M, \widetilde{g})$ of $\bar{M}$ are

$$
\begin{aligned}
& \widetilde{\widetilde{\nabla}}_{X} Y=\widetilde{\nabla}_{X} Y+\widetilde{h}(X, Y), \\
& \widetilde{\nabla}_{X} V=-\widetilde{A}_{V} X+\nabla_{X}^{t} V,
\end{aligned}
$$

where: $\left\{\widetilde{\nabla}_{X} Y, \widetilde{A}_{V} X\right\}$ and $\left\{\widetilde{h}(X, Y), \nabla_{X}^{t} V\right\}$ belong to $\Gamma(T M)$ and $\Gamma(\operatorname{tr}(T M))$, respectively; $\widetilde{\nabla}$ and $\nabla^{t}$ are linear connections on $(M, \widetilde{g})$ and $\operatorname{tr}(T M)$, respectively. Moreover, $\widetilde{\nabla}$ is torsion-free linear connection, $\widetilde{h}$ is a $\Gamma(\operatorname{tr}(T M))$-valued symmetric $\mathcal{F}(M)$-bilinear form on $\Gamma(T M)$ and $\widetilde{A}$ is a $\Gamma(T M)$-valued $\mathcal{F}(M)$-bilinear form on $\Gamma(\operatorname{tr}(T M)) \times \Gamma(T M)$. In general, $\widetilde{\nabla}$ and $\nabla^{t}$ are not metric connections. In ${ }^{1}$ the following objects are introduced

$$
\begin{array}{ll}
h^{l}(X, Y)=L(\widetilde{h}(X, Y)) ; & h^{s}(X, Y)=S(\widetilde{h}(X, Y)) ; \\
D_{X}^{l} V=L\left(\nabla_{X}^{t} V\right) ; & D_{X}^{s} V=S\left(\nabla_{X}^{t} V\right),
\end{array}
$$

where $L$ and $S$ are the projection morphisms of $\operatorname{tr}(T M)$ on $\operatorname{ltr}(T M)$ and $S\left(T M^{\widetilde{\perp}}\right)$, respectively. Then the formulas (31) become

$$
\begin{aligned}
& \widetilde{\nabla}_{X} Y=\widetilde{\nabla}_{X} Y+h^{l}(X, Y)+h^{s}(X, Y), \\
& \widetilde{\nabla}_{X} V=-\widetilde{A}_{V} X+D_{X}^{l} V+D_{X}^{s} V .
\end{aligned}
$$


Besides $D^{l}$ and $D^{s}$ do not define linear connections on $\operatorname{tr}(T M)$ but they are Otsuki connections on $\operatorname{tr}(T M)$ with respect to $L$ and $S$, respectively. We note that for an $r$-lightlike submanifold $D^{l}$ is a metric Otsuki connection on $\operatorname{tr}(T M)$. Now, the formulas of Gauss and Weingarten for an isotropic submanifold $(M, \widetilde{g})$ of $\bar{M}$ are

$$
\begin{aligned}
& \widetilde{\widetilde{\nabla}}_{X} Y=\widetilde{\nabla}_{X} Y+h^{s}(X, Y), \\
& \widetilde{\nabla}_{X} N=-\widetilde{A}_{N} X+\nabla_{X}^{l} N+\mathcal{D}^{s}(X, N), \\
& \widetilde{\nabla}_{X} W=-\widetilde{A}_{W} X+\mathcal{D}^{l}(X, W)+\nabla_{X}^{s} W,
\end{aligned}
$$

where: $\widetilde{\nabla}$ is a metric linear connection on $(M, \widetilde{g}) ; \nabla^{l}$ and $\nabla^{s}$, defined by $\nabla_{X}^{l} N=D_{X}^{l} N$ and $\nabla_{X}^{s} W=D_{X}^{s} W$, are metric linear connections on $\operatorname{ltr}(T M)$ and $S\left(T M^{\widetilde{\perp}}\right)$, respectively; $\mathcal{D}^{l}$ and $\mathcal{D}^{s}$, defined by $\mathcal{D}^{l}(X, W)=$ $D_{X}^{l} W$ and $\mathcal{D}^{s}(X, N)=D_{X}^{s} N$, are $\mathcal{F}(\bar{M})$-bilinear mappings. By using (32) and taking into account that $\bar{\nabla}$ is a metric connection we obtain

$$
\begin{aligned}
& \overline{\widetilde{g}}\left(h^{s}(X, Y), W\right)+\overline{\widetilde{g}}\left(Y, \mathcal{D}^{l}(X, W)\right)=0 ; \quad \overline{\widetilde{g}}\left(\mathcal{D}^{s}(X, N), W\right)=\overline{\widetilde{g}}\left(N, \widetilde{A}_{W} X\right) \\
& \overline{\widetilde{g}}\left(\widetilde{A}_{N} X, N^{\prime}\right)+\overline{\widetilde{g}}\left(\widetilde{A}_{N^{\prime}} X, N\right)=0 .
\end{aligned}
$$

Further, we will find the formulas of Gauss and Weingarten for $(M, g)$. Denoting by $\bar{\nabla}$ and $\nabla$ the Levi-Civita connections of $\bar{g}$ and $g$ on $\bar{M}$ and $(M, g)$ respectively, we have

$$
\begin{aligned}
& \bar{\nabla}_{X} Y=\nabla_{X} Y+h(X, Y), \\
& \bar{\nabla}_{X} V=-A_{V} X+D_{X} V,
\end{aligned}
$$

where: $h$ is the second fundamental form of $(M, g) ; A_{V}$ is the shape operator of $(M, g)$ with respect to $V ; D$ is the normal connection on $T M^{\perp}$ which is a metric linear connection. Taking into account the first equality of (30), the formulas (33) become

$$
\begin{aligned}
& \bar{\nabla}_{X} Y=\nabla_{X} Y+h^{1}(X, Y)+h^{2}(X, Y), \\
& \bar{\nabla}_{X} V=-A_{V} X+D_{X}^{1} V+D_{X}^{2} V,
\end{aligned}
$$

where we denote

$$
\begin{array}{ll}
h^{1}(X, Y)=P_{1}(h(X, Y)) ; & h^{2}(X, Y)=P_{2}(h(X, Y)) ; \\
D_{X}^{1} V=P_{1}\left(D_{X} V\right) ; & D_{X}^{2} V=P_{2}\left(D_{X} V\right)
\end{array}
$$

and $P_{1}, P_{2}$ are the projection morphisms of $T M^{\perp}$ on $\bar{J} T M,(\bar{J} T M)^{\perp}$, respectively. By direct calculations we check that $D^{1}$ and $D^{2}$ do not define linear connections on $T M^{\perp}$ but both are Otsuki connections with respect 
to the vector bundle morphism $P_{1}$ and $P_{2}$, respectively. So, the formulas (34) can be written as follows

$$
\begin{aligned}
& \bar{\nabla}_{X} Y=\nabla_{X} Y+h^{1}(X, Y)+h^{2}(X, Y), \\
& \bar{\nabla}_{X} N=-A_{N} X+\nabla_{X}^{1} N+\mathcal{D}^{2}(X, N), \\
& \bar{\nabla}_{X} W=-A_{W} X+\mathcal{D}^{1}(X, W)+\nabla_{X}^{2} W,
\end{aligned}
$$

where: $\nabla^{1}$ and $\nabla^{2}$, defined by $\nabla_{X}^{1} N=D_{X}^{1} N$ and $\nabla_{X}^{2} W=D_{X}^{2} W$, are metric linear connections on $\bar{J} T M$ and $(\bar{J} T M)^{\perp}$, respectively; $\mathcal{D}^{1}$ and $\mathcal{D}^{2}$, defined by $\mathcal{D}^{1}(X, W)=D_{X}^{1} W$ and $\mathcal{D}^{2}(X, N)=D_{X}^{2} N$, are $\mathcal{F}(\bar{M})$-bilinear mappings. As $\bar{\nabla}$ is a metric connection, from (35) we obtain

$$
\begin{gathered}
\bar{g}\left(h^{1}(X, Y), N\right)=\bar{g}\left(A_{N} X, Y\right), \\
\bar{g}\left(h^{2}(X, Y), W\right)=\bar{g}\left(A_{W} X, Y\right), \\
\bar{g}\left(\mathcal{D}^{2}(X, N), W\right)=-\bar{g}\left(\mathcal{D}^{1}(X, W), N\right) .
\end{gathered}
$$

Theorem 4.1. Let $(M, g)$ be a totally real submanifold of $(\bar{M}, \bar{J}, \bar{g}, \overline{\widetilde{g}})$. Then the following assertions are equivalent:

(i) $D^{1}$ is a metric Otsuki connection on $T M^{\perp}$.

(ii) $\mathcal{D}^{1}(X, W)=0$, for any $X \in \Gamma(T M), W \in \Gamma\left((\bar{J} T M)^{\perp}\right)$.

(iii) $\mathcal{D}^{2}(X, N)=0$, for any $X \in \Gamma(T M), N \in \Gamma(\bar{J} T M)$.

(iv) $D^{2}$ is a metric Otsuki connection on $T M^{\perp}$.

Proof. Using that $\nabla^{1}$ and $\nabla^{2}$ are metric linear connections on $\bar{J} T M$ and $(\bar{J} T M)^{\perp}$ respectively and $(38)$ we have

$\left(D_{X}^{1} \bar{g}\right)\left(N, N^{\prime}\right)=\left(D_{X}^{2} \bar{g}\right)\left(N, N^{\prime}\right)=0,\left(D_{X}^{1} \bar{g}\right)\left(W, W^{\prime}\right)=\left(D_{X}^{2} \bar{g}\right)\left(W, W^{\prime}\right)=0$, $\left(D_{X}^{1} \bar{g}\right)(W, N)=-\bar{g}\left(\mathcal{D}^{1}(X, W), N\right)=\bar{g}\left(\mathcal{D}^{2}(X, N), W\right)=-\left(D_{X}^{2} \bar{g}\right)(W, N)$,

In this way we see that assertions $(i),(i i),(i i i)$ and $(i v)$ are equivalent.

According to (30), we can write (5) in the following manner

$$
\overline{\widetilde{\nabla}}_{\bar{X}} \bar{Y}=\bar{\nabla}_{\bar{X}} \bar{Y}+\Phi^{\prime}(\bar{X}, \bar{Y})+\Phi^{1}(\bar{X}, \bar{Y})+\Phi^{2}(\bar{X}, \bar{Y})
$$

where we denote by $\Phi^{\prime}(\bar{X}, \bar{Y}), \Phi^{1}(\bar{X}, \bar{Y})$ and $\Phi^{2}(\bar{X}, \bar{Y})$ the part of $\Phi(\bar{X}, \bar{Y})$ which belongs to $\Gamma(T M), \Gamma(\bar{J} T M)=\Gamma(\operatorname{ltr}(T M))$ and $\Gamma\left((\bar{J} T M)^{\perp}\right)=$ 
$\Gamma\left(S\left(T M^{\widetilde{\perp}}\right)\right)$, respectively. By using (32) and (35) from (39) we get

$$
\begin{aligned}
\widetilde{\nabla}_{X} Y+h^{s}(X, Y)= & \nabla_{X} Y+h^{1}(X, Y)+h^{2}(X, Y) \\
& +\Phi^{\prime}(X, Y)+\Phi^{1}(X, Y)+\Phi^{2}(X, Y) \\
-\widetilde{A}_{N} X+\nabla_{X}^{l} N+\mathcal{D}^{s}(X, N) & =-A_{N} X+\nabla_{X}^{1} N+\mathcal{D}^{2}(X, N) \\
& +\Phi^{\prime}(X, N)+\Phi^{1}(X, N)+\Phi^{2}(X, N) \\
-\widetilde{A}_{W} X+\mathcal{D}^{l}(X, W)+\nabla_{X}^{s} W & =-A_{W} X+\mathcal{D}^{1}(X, W)+\nabla_{X}^{2} W \\
& +\Phi^{\prime}(X, W)+\Phi^{1}(X, W)+\Phi^{2}(X, W) .
\end{aligned}
$$

Comparing the parts belonging to $\Gamma(T M), \Gamma(\bar{J} T M)=\Gamma(\operatorname{ltr}(T M))$ and $\Gamma\left((\bar{J} T M)^{\perp}\right)=\Gamma\left(S\left(T M^{\widetilde{\perp}}\right)\right)$ of both sides of the above equations, we obtain the following relations

$$
\begin{array}{ll}
\widetilde{\nabla}_{X} Y=\nabla_{X} Y+\Phi^{\prime}(X, Y), & h^{s}(X, Y)=h^{2}(X, Y)+\Phi^{2}(X, Y), \\
h^{1}(X, Y)=-\Phi^{1}(X, Y), & \widetilde{A}_{N} X=A_{N} X-\Phi^{\prime}(X, N), \\
\nabla_{X}^{l} N=\nabla_{X}^{1} N+\Phi^{1}(X, N), \quad \mathcal{D}^{s}(X, N)=\mathcal{D}^{2}(X, N)+\Phi^{2}(X, N), \\
\widetilde{A}_{W} X=A_{W} X-\Phi^{\prime}(X, W), & \mathcal{D}^{l}(X, W)=\mathcal{D}^{1}(X, W)+\Phi^{1}(X, W), \\
\nabla_{X}^{s} W=\nabla_{X}^{2} W+\Phi^{2}(X, W) . &
\end{array}
$$

In ${ }^{3}$ the eight classes of almost complex manifolds with Norden metric are characterized by conditions for the tensor $F$. In ${ }^{4}$ the following relations between the tensor $F$ and $\Phi$ are obtained

$$
\begin{gathered}
\Phi(\bar{X}, \bar{Y}, \bar{Z})=\frac{1}{2}(F(\overline{J Z}, \bar{X}, \bar{Y})-F(\bar{X}, \bar{Y}, \bar{J} Z)-F(\bar{Y}, \bar{J} Z, \bar{X})), \\
F(\bar{X}, \bar{Y}, \bar{Z})=\Phi(\bar{X}, \bar{Y}, \overline{J Z})+\Phi(\bar{X}, \bar{Z}, \overline{J Y}),
\end{gathered}
$$

where $\Phi(\bar{X}, \bar{Y}, \bar{Z})=\bar{g}(\Phi(\bar{X}, \bar{Y}), \bar{Z})$. Also in ${ }^{4}$, by using (41) and (42), every basic class of almost complex manifolds with Norden metric is characterized by conditions for the tensor $\Phi$. Taking into account both types of characterization conditions, we can specify the equations (40) when the ambient manifold $\bar{M}$ belongs to any basic class. Further, in this section we consider only the case when $\bar{M}$ is a Kaehler manifold with Norden metric. Then the characterization condition of $\bar{M}$ is $F(\bar{X}, \bar{Y}, \bar{Z})=0$ which is equivalent to $\left(\bar{\nabla}_{\bar{X}} \bar{J}\right) \bar{Y}=0$.

Lemma 4.1. Let $\bar{M}$ be a Kaehler manifold with Norden metric and $(M, g)$ be a totally real submanifold of $\bar{M}$. Then

$$
\begin{gathered}
h^{1}(X, Y)=0, \quad A_{\bar{J} Y} X=0, \\
\nabla_{X}^{1} \bar{J} Y=\bar{J}\left(\nabla_{X} Y\right),
\end{gathered}
$$




$$
\begin{gathered}
\mathcal{D}^{2}(X, \bar{J} Y)=\bar{J}\left(h^{2}(X, Y)\right), \\
A_{W} X=\bar{J}\left(\mathcal{D}^{1}(X, \bar{J} W)\right), \\
\nabla_{X}^{2} \bar{J} W=\bar{J}\left(\nabla_{X}^{2} W\right) .
\end{gathered}
$$

Proof. From $\bar{\nabla}_{\bar{X}} \bar{J} Y=\bar{J} \overline{ }_{\bar{X}} \bar{Y}$ and (35) we obtain the relations (44) $\div$ (47) and $h^{1}(X, Y)=\bar{J} A_{\bar{J} Y} X$. On the other hand, replacing $N$ by $\bar{J} Y$ in (36) we have $h^{1}(X, Y)=-\bar{J} A_{\bar{J} Y} X$. Hence, (43) is fulfilled.

The characterization condition $F=0$ for a Kaehler manifold with Norden $\bar{M}$ and (41) imply that $\Phi=0$ for $\bar{M}$. Then the formulas (5) and (40) become

$$
\bar{\nabla}_{X} Y=\overline{\widetilde{\nabla}}_{X} Y
$$

and

$$
\begin{aligned}
& \widetilde{\nabla}_{X} Y=\nabla_{X} Y, \quad h^{s}(X, Y)=h^{2}(X, Y), \quad h^{1}(X, Y)=0, \\
& \widetilde{A}_{N} X=A_{N} X, \quad \nabla_{X}^{l} N=\nabla_{X}^{1} N, \quad \mathcal{D}^{s}(X, N)=\mathcal{D}^{2}(X, N), \\
& \widetilde{A}_{W} X=A_{W} X, \quad \mathcal{D}^{l}(X, W)=\mathcal{D}^{1}(X, W), \quad \nabla_{X}^{s} W=\nabla_{X}^{2} W .
\end{aligned}
$$

From (49) it is clear that the relations $(43) \div(47)$ are valid if we replace $\nabla, h^{1}, h^{2}, A_{N}, A_{W}, \nabla^{1}, \nabla^{2}, \mathcal{D}^{1}, \mathcal{D}^{2}$ by $\widetilde{\nabla}, h^{l}, h^{s}, \widetilde{A}_{N}, \widetilde{A}_{W}, \nabla^{l}, \nabla^{s}, \mathcal{D}^{l}, \mathcal{D}^{s}$, respectively.

Theorem 4.2. Let $\bar{M}$ be a Kaehler manifold with Norden metric and $(M, g)$ be a totally real submanifold of $\bar{M}$. Then the following assertions are equivalent:

(i) $(M, g)$ is totally geodesic.

(ii) $h^{2}$ vanishes identically on $(M, g)$.

(iii) $A_{W}$ vanishes identically on $(M, g)$.

(iv) $\mathcal{D}^{1}(X, W)=0$, for $\forall X \in \Gamma(T M), \forall W \in \Gamma\left((\bar{J} T M)^{\perp}\right)$.

(v) $\mathcal{D}^{2}(X, N)=0$, for $\forall X \in \Gamma(T M), \forall N \in \Gamma(\bar{J} T M)$.

Proof. The equality (43) implies $h(X, Y)=h^{2}(X, Y)$ and consequently $(i) \Longleftrightarrow(i i)$. The equivalence of $(i i)$ and $($ iii $)$ follows from (37). Because of (45) and (46) we have $(i i) \Longleftrightarrow(v)$ and $(i i i) \Longleftrightarrow(i v)$, respectively.

Theorem 4.3. Let $\bar{M}$ be a Kaehler manifold with Norden metric. Then the following assertions are equivalent:

(i) $(M, g)$ is totally geodesic. 
(ii) $(M, \widetilde{g})$ is totally geodesic.

(iii) $D^{1}$ is a metric Otsuki connection on $T M^{\perp}$.

(iv) $D^{2}$ is a metric Otsuki connection on $T M^{\perp}$.

(v) $\nabla^{t}$ is a metric linear connection on $\operatorname{tr}(T M)$.

(vi) $D^{s}$ is a metric Otsuki connection on $\operatorname{tr}(T M)$.

Proof. According to Theorem 4.1 and Theorem 4.2, each from the assertions $(i),(i i i),(i v)$ is equivalent to the condition $\mathcal{D}^{2}(X, N)=0 . \mathrm{In}^{1}$ (page 159 and 167) it is proved that assertions $(v),(v i)$ and (ii) are equivalent to $\mathcal{D}^{s}(X, N)=0$ and $h^{s}(X, Y)=0$, respectively. From (48) we have $\mathcal{D}^{2}(X, N)=\mathcal{D}^{s}(X, N)=0$ and $h^{2}(X, Y)=h^{s}(X, Y)=0$. Finally, according to Theorem 4.2 the conditions $\mathcal{D}^{2}(X, N)=0$ and $h^{2}(X, Y)=0$ are equivalent which completes the proof.

Structure equations of the submanifolds $(M, g)$ and $(M, \widetilde{g})$.

Denote by $\bar{R}, R$ and $R^{\perp}$ the curvature tensors of type $(1,3)$ of $\bar{\nabla}, \nabla$ and $D$, respectively. We define the curvature tensors $\left(R^{\perp}\right)^{1}$ and $\left(R^{\perp}\right)^{2}$ of $\nabla^{1}$ and $\nabla^{2}$ by $\left(R^{\perp}\right)^{1}(X, Y, N)=\nabla_{X}^{1} \nabla_{Y}^{1} N-\nabla_{Y}^{1} \nabla_{X}^{1} N-\nabla_{[X, Y]}^{1} N$ and $\left(R^{\perp}\right)^{2}(X, Y, W)=\nabla_{X}^{2} \nabla_{Y}^{2} W-\nabla_{Y}^{2} \nabla_{X}^{2} W-\nabla_{[X, Y]}^{2} W$. Then by straightforward calculations using (35) and Lemma 4.1 we obtain

$$
\begin{aligned}
\bar{R}(X, Y, Z) & =R(X, Y, Z)-A\left(h^{2}(Y, Z), X\right)+A\left(h^{2}(X, Z), Y\right) \\
& +\bar{J}\left(A\left(\bar{J} h^{2}(Y, Z), X\right)-A\left(\bar{J} h^{2}(X, Z), Y\right)\right) \\
& +\left(\nabla_{X} h^{2}\right)(Y, Z)-\left(\nabla_{Y} h^{2}\right)(X, Z), \\
\bar{R}(X, Y, W)= & \left(R^{\perp}\right)^{2}(X, Y, W)-h^{2}\left(X, A_{W} Y\right)+h^{2}\left(Y, A_{W} X\right) \\
+ & \bar{J}\left(h^{2}\left(X, A_{\bar{J}} W\right)-h^{2}\left(Y, A_{\bar{J} W} X\right)\right)-\left(\nabla_{X} A\right)(W, Y) \\
+ & \left(\nabla_{Y} A\right)(W, X)+\bar{J}\left(\left(\nabla_{X} A\right)(\bar{J} W, Y)-\left(\nabla_{Y} A\right)(\bar{J} W, X)\right),
\end{aligned}
$$

where

$$
\begin{aligned}
& \left(\nabla_{X} h^{2}\right)(Y, Z)=\nabla_{X}^{2} h^{2}(Y, Z)-h^{2}\left(\nabla_{X} Y, Z\right)-h^{2}\left(Y, \nabla_{X} Z\right) \\
& \left(\nabla_{X} A\right)(W, Y)=\nabla_{X} A(W, Y)-A\left(\nabla_{X}^{2} W, Y\right)-A\left(W, \nabla_{X} Y\right) .
\end{aligned}
$$

For convenience we denote $A_{V} X$ by $A(V, X)$ where it is necessary. The equality $\bar{\nabla}_{\bar{X}} \bar{J} Y=\bar{J}_{\bar{X}} \bar{Y}$ implies

$$
\bar{R}(\bar{X}, \bar{Y}, \overline{J Z})=\overline{J R}(\bar{X}, \bar{Y}, \bar{Z}) .
$$

Hence, from (50) we have

$$
\begin{gathered}
\bar{R}(X, Y, \bar{J} Z)=\bar{J} R(X, Y, Z)-\bar{J}\left(A\left(h^{2}(Y, Z), X\right)-A\left(h^{2}(X, Z), Y\right)\right) \\
-A\left(\bar{J} h^{2}(Y, Z), X\right)+A\left(\bar{J} h^{2}(X, Z), Y\right) \\
+\bar{J}\left(\left(\nabla_{X} h^{2}\right)(Y, Z)-\left(\nabla_{Y} h^{2}\right)(X, Z)\right) .
\end{gathered}
$$


Now, using $D_{X} N=D_{X}^{1} N+D_{X}^{2} N=\nabla_{X}^{1} N+\mathcal{D}^{2}(X, N), \quad D_{X} W=D_{X}^{1} W+$ $D_{X}^{2} W=\mathcal{D}^{1}(X, W)+\nabla_{X}^{2} W$ and Lemma 4.1 we compute

$$
\begin{aligned}
R^{\perp}(X, Y, \bar{J} Z) & =D_{X} D_{Y} \bar{J} Z-D_{Y} D_{X} \bar{J} Z-D_{[X, Y]} \bar{J} Z \\
& =\bar{J} R(X, Y, Z)+\bar{J}\left(\left(\nabla_{X} h^{2}\right)(Y, Z)-\left(\nabla_{Y} h^{2}\right)(X, Z)\right) \\
& -\bar{J}\left(A\left(h^{2}(Y, Z), X\right)-A\left(h^{2}(X, Z), Y\right)\right), \\
R^{\perp}(X, Y, W) & =D_{X} D_{Y} W-D_{Y} D_{X} W-D_{[X, Y]} W \\
& =\left(R^{\perp}\right)^{2}(X, Y, W)+\bar{J}\left(\left(\nabla_{X} A\right)(\bar{J} W, Y)-\left(\nabla_{Y} A\right)(\bar{J} W, X)\right) \\
& -\bar{J}\left(h^{2}(Y, A(\bar{J} W, X))-h^{2}(X, A(\bar{J} W, Y))\right) .
\end{aligned}
$$

From (48) and (49) it follows $\bar{R}=\overline{\widetilde{R}}, R=\widetilde{R},\left(R^{\perp}\right)^{1}=R^{l},\left(R^{\perp}\right)^{2}=$ $R^{s}$. Thus the equalities (50), (51), (52), (53) are valid if we replace $\bar{R}, R,\left(R^{\perp}\right)^{2}, A_{W}, h^{2}$ by $\widetilde{R}, \widetilde{R}, R^{s}, \widetilde{A}_{W}, h^{s}$, respectively. We note that $\widetilde{\widetilde{R}}(X, Y, Z), \widetilde{R}(X, Y, \bar{J} Z), \overline{\widetilde{R}}(X, Y, W)$ can be obtained from ${ }^{1}$ (page 175) by using (49) and Lemma 4.1. Taking into account (44) and (47) we have

$$
\bar{J} R(X, Y, Z)=\left(R^{\perp}\right)^{1}(X, Y, \bar{J} Z)=R^{l}(X, Y, \bar{J} Z)=\bar{J} \widetilde{R}(X, Y, Z)
$$

and

$$
\bar{J}\left(R^{\perp}\right)^{2}(X, Y, W)=\left(R^{\perp}\right)^{2}(X, Y, \bar{J} W)=R^{s}(X, Y, \bar{J} W)=\bar{J} R^{s}(X, Y, W) .
$$

It is known that the Levi-Civita connection $\bar{\nabla}$ (resp. $\bar{\nabla}$ ) is said to be flat if $\bar{R}=0$ (resp. $\overline{\widetilde{R}}=0$ ). Also, if $R^{\perp}=0$ then the normal connection $D$ is said to be flat. Analogously, we say that $\nabla^{1}, \nabla^{2}, \nabla^{l}, \nabla^{s}$ are flat if the corresponding curvature tensors $\left(R^{\perp}\right)^{1},\left(R^{\perp}\right)^{2}, R^{l}, R^{s}$ are equal to zero. As a corollary of (56) we state

Proposition 4.1. For the submanifolds $(M, g)$ and $(M, \widetilde{g})$ of a Kaehler manifold with Norden metric $\bar{M}$ the following assertions are equivalent: 1) $\nabla$ is flat; 2) $\widetilde{\nabla}$ is flat; 3$) \nabla^{1}$ is flat; 4$) \nabla^{l}$ is flat.

From (50) we obtain the equation of Gauss and Codazzi

$\bar{R}(X, Y, Z, U)=R(X, Y, Z, U)-\bar{g}\left(A\left(h^{2}(Y, Z), X\right), U\right)+\bar{g}\left(A\left(h^{2}(X, Z), Y\right), U\right)$

and

$$
\begin{aligned}
(\bar{R}(X, Y, Z))^{\perp} & =\left(\nabla_{X} h^{2}\right)(Y, Z)-\left(\nabla_{Y} h^{2}\right)(X, Z) \\
& +\bar{J}\left(A\left(\bar{J} h^{2}(Y, Z), X\right)-A\left(\bar{J} h^{2}(X, Z), Y\right)\right) .
\end{aligned}
$$

The equality (52) and the fact that $\bar{J}$ is an anti-isometry with respect to $\bar{g}$ imply $\bar{R}(\bar{X}, \bar{Y}, \bar{Z}, \bar{U})=\bar{g}(\bar{R}(\bar{X}, \bar{Y}, \bar{Z}), \bar{U})$ is a Kaehler tensor, i.e.

$$
\bar{R}(\bar{X}, \bar{Y}, \overline{J Z}, \overline{J U})=-\bar{R}(\bar{X}, \bar{Y}, \bar{Z}, \bar{U}) .
$$


Using (57), (54), (55) and (37) for the equation of Ricci we get

$\bar{g}(\bar{R}(X, Y, \bar{J} Z), \bar{J} U)=-\bar{R}(X, Y, Z, U)$, $\bar{g}(\bar{R}(X, Y, \bar{J} Z), W)=\bar{g}\left(\left(\nabla_{X} h^{2}\right)(Y, Z)-\left(\nabla_{Y} h^{2}\right)(X, Z), \bar{J} W\right)$, $\bar{g}\left(\bar{R}(X, Y, W), W^{\prime}\right)=\bar{g}\left(\left(R^{\perp}\right)^{2}(X, Y, W), W^{\prime}\right)-\bar{g}\left(A\left(\bar{J} W^{\prime}, Y\right), A(\bar{J} W, X)\right)$ $+\bar{g}\left(A\left(\bar{J} W^{\prime}, X\right), A(\bar{J} W, Y)\right)+\bar{g}\left(A_{W} X, A_{W^{\prime}} Y\right)-\bar{g}\left(A_{W^{\prime}} X, A_{W} Y\right)$.

For the curvature tensor $\overline{\widetilde{R}}$ of type $(0,4)$ we have

$$
\overline{\widetilde{R}}(\bar{X}, \bar{Y}, \bar{Z}, \bar{U})=\overline{\widetilde{g}}(\overline{\widetilde{R}}(\bar{X}, \bar{Y}, \bar{Z}), \bar{U})=\bar{R}(\bar{X}, \bar{Y}, \bar{Z}, \overline{J U}) .
$$

By direct calculations we check that $\overline{\widetilde{R}}$ is a Kaehler tensor, too. The structure equations of $\overline{\widetilde{R}}$ we obtain using (37), (49), (58) and equations of Gauss, Codazzi and Ricci

$$
\begin{aligned}
& \overline{\widetilde{R}}(X, Y, Z, N)=\overline{\widetilde{g}}(\widetilde{R}(X, Y, Z), N)-\overline{\widetilde{g}}\left(\widetilde{A}\left(h^{s}(Y, Z), X\right), N\right) \\
& +\overline{\widetilde{g}}\left(\widetilde{A}\left(h^{s}(X, Z), Y\right), N\right), \\
& \overline{\widetilde{R}}\left(X, Y, N^{\prime}, N\right)=\overline{\widetilde{g}}\left(\widetilde{A}\left(\bar{J} h^{s}(Y, \bar{J} N), X\right), N^{\prime}\right)-\overline{\widetilde{g}}\left(\widetilde{A}\left(\bar{J} h^{s}(X, \bar{J} N), Y\right), N^{\prime}\right), \\
& \overline{\widetilde{R}}(X, Y, W, N)=\overline{\widetilde{g}}\left(\left(\widetilde{\nabla}_{Y} \widetilde{A}\right)(W, X), N\right)-\overline{\widetilde{g}}\left(\left(\widetilde{\nabla}_{X} \widetilde{A}\right)(W, Y), N\right), \\
& \overline{\widetilde{R}}(X, Y, Z, U)=\overline{\widetilde{g}}\left(h^{s}(Y, U), h^{s}(X, Z)\right)-\overline{\widetilde{g}}\left(h^{s}(X, U), h^{s}(Y, Z)\right), \\
& \overline{\widetilde{R}}(X, Y, W, U)=\overline{\widetilde{g}}\left(\left(\widetilde{\nabla}_{Y} h^{s}\right)(X, U), W\right)-\overline{\widetilde{g}}\left(\left(\widetilde{\nabla}_{X} h^{s}\right)(Y, U), W\right), \\
& \overline{\widetilde{R}}\left(X, Y, W^{\prime}, W\right)=\overline{\widetilde{g}}\left(R^{s}\left(X, Y, W^{\prime}\right), W\right)-\overline{\widetilde{g}}\left(\widetilde{A}_{W} Y, \bar{J} \widetilde{A}_{\bar{J} W^{\prime}}, X\right) \\
& +\overline{\widetilde{g}}\left(\widetilde{A}_{W} X, \bar{J} \widetilde{A}_{\bar{J}_{W^{\prime}}} Y\right)-\overline{\widetilde{g}}\left(\widetilde{A}_{W^{\prime}} X, \bar{J} \widetilde{A}_{\bar{J} W} Y\right)+\overline{\widetilde{g}}\left(\widetilde{A}_{W^{\prime}} Y, \bar{J} \widetilde{A}_{\bar{J} W} X\right), \\
& \overline{\widetilde{g}}(\widetilde{R}(X, Y, Z), N)+\overline{\widetilde{g}}\left(R^{l}(X, Y, N), Z\right)=0 \text {. }
\end{aligned}
$$

\section{Examples}

According to the obtained results in Section 3, each from the constructed examples below is an example for a submanifold of an almost complex manifold with Norden metric which is non-degenerate with respect to the one Norden metric and lightlike with respect to the other.

Example 5.1. We consider the Lie group $G L(2 ; \mathbb{R})$ with a Lie algebra $\operatorname{gl}(2 ; \mathbb{R})$. The real Lie algebra $g l(2 ; \mathbb{R})$ is spanned by the left invariant vector fields $\left\{X_{1}, X_{2}, X_{3}, X_{4}\right\}$ where we set

$$
X_{1}=\left(\begin{array}{ll}
1 & 0 \\
0 & 0
\end{array}\right), X_{2}=\left(\begin{array}{ll}
0 & 1 \\
0 & 0
\end{array}\right), X_{3}=\left(\begin{array}{ll}
0 & 0 \\
1 & 0
\end{array}\right), X_{4}=\left(\begin{array}{ll}
0 & 0 \\
0 & 1
\end{array}\right) .
$$


We define an almost complex structure $\bar{J}$ and a left invariant metric $\bar{g}$ on $g l(2 ; \mathbb{R})$ by

$$
\bar{J} X_{1}=X_{4}, \quad \bar{J} X_{2}=X_{3}, \quad \bar{J} X_{3}=-X_{2}, \quad \bar{J} X_{4}=-X_{1}
$$

and

$$
\begin{aligned}
& \bar{g}\left(X_{i}, X_{i}\right)=-\bar{g}\left(X_{j}, X_{j}\right)=-1 ; \quad i=1,3 ; \quad j=2,4 \\
& \bar{g}\left(X_{i}, X_{j}\right)=0 ; \quad i \neq j ; \quad i, j=1,2,3,4 .
\end{aligned}
$$

Using (59) and (60) we check that the metric $\bar{g}$ is a Norden metric and consequently $(G L(2 ; \mathbb{R}), \bar{J}, \bar{g}, \overline{\widetilde{g}})$ is a 4 -dimensional almost complex manifold with Norden metric. The real special linear group $S L(2 ; \mathbb{R})=\{A \in$ $G L(2 ; \mathbb{R}): \operatorname{det}(A)=1\}$ is a Lie subgroup of $G L(2 ; \mathbb{R})$ with a Lie algebra $\operatorname{sl}(2 ; \mathbb{R})$ of all $(2 \times 2)$ real traceless matrices. Thus $S L(2 ; \mathbb{R})$ is a 3 dimensional submanifold of $G L(2 ; \mathbb{R})$ and $\operatorname{sl}(2 ; \mathbb{R})$ is a subalgebra of $g l(2 ; \mathbb{R})$ spanned by $\left\{X_{1}-X_{4}, X_{2}, X_{3}\right\}$. We find that the normal space $\operatorname{sl}(2 ; \mathbb{R})^{\perp}$ is spanned by $\left\{X_{1}, X_{4}\right\}$. Hence $\operatorname{sl}(2 ; \mathbb{R}) \cap \operatorname{sl}(2 ; \mathbb{R})^{\perp}=\left\{X_{1}-X_{4}\right\}$, i.e. $(S L(2 ; \mathbb{R}), g)$ is an 1 -dimensional lightlike submanifold with $\operatorname{Rad} s l(2 ; \mathbb{R})=$ $\operatorname{span}\left\{\xi=\frac{X_{1}-X_{4}}{\sqrt{2}}\right\}$. As $\operatorname{dimRad} s l(2 ; \mathbb{R})=\operatorname{codim} S L(2 ; \mathbb{R})=1$ it follows that $(S L(2 ; \mathbb{R}), g)$ is a coisotropic submanifold of $G L(2 ; \mathbb{R})$. The screen distribution $S(\operatorname{sl}(2 ; \mathbb{R}))$ is spanned by $\left\{X_{2}, X_{3}=\bar{J} X_{2}\right\}$ which means that $S(\operatorname{sl}(2 ; \mathbb{R}))$ is holomorphic with respect to $\bar{J}$. Choose $\operatorname{sl}(2 ; \mathbb{R})^{\perp}=$ $\operatorname{span}\left\{\xi, \frac{-X_{1}-X_{4}}{\sqrt{2}}\right\}$ we have $\operatorname{ltr}(\operatorname{sl}(2 ; \mathbb{R}))=\operatorname{span}\left\{\frac{-X_{1}-X_{4}}{\sqrt{2}}\right\}$. Using (59) we check that $\bar{J} \operatorname{Rad} s l(2 ; \mathbb{R})=\bar{J} \xi=\operatorname{ltr}(\operatorname{sl}(2 ; \mathbb{R}))$. Thus $(S L(2 ; \mathbb{R}), g)$ is a coisotropic Radical transversal lightlike submanifold of $G L(2 ; \mathbb{R})$ and from Corollary 3.1 it follows that $(S L(2 ; \mathbb{R}), \widetilde{g})$ is a generic submanifold.

Now, we consider the Lie group $G L(2 ; \mathbb{C})$ with its real 8-dimensional Lie algebra $g l(2 ; \mathbb{C})$. We define a complex structure $\bar{J}$ on $g l(2 ; \mathbb{C})$ by $\overline{J X}=i \bar{X}$ for any left invariant vector field $\bar{X} \in g l(2 ; \mathbb{C})$. Hence we have $[\overline{J X}, \bar{Y}]=$ $\bar{J}[\bar{X}, \bar{Y}]$, i.e. $\bar{J}$ is a bi-invariant complex structure. The Lie algebra $g l(2 ; \mathbb{C})$ is spanned by the left invariant vector fields $\left\{X_{1}, X_{2}, \ldots, X_{8}\right\}$ where we set

$$
X_{1}=\left(\begin{array}{ll}
1 & 0 \\
0 & 0
\end{array}\right), X_{3}=\left(\begin{array}{ll}
0 & 1 \\
0 & 0
\end{array}\right), X_{5}=\left(\begin{array}{ll}
0 & 0 \\
1 & 0
\end{array}\right), X_{7}=\left(\begin{array}{ll}
0 & 0 \\
0 & 1
\end{array}\right),
$$

$X_{2}=\bar{J} X_{1}, \quad X_{4}=\bar{J} X_{3}, \quad X_{6}=\bar{J} X_{5}, \quad X_{8}=\bar{J} X_{7}$

We define a left invariant metric $\bar{g}$ on $g l(2 ; \mathbb{C})$ by

$$
\begin{aligned}
& \bar{g}\left(X_{i}, X_{i}\right)=-\bar{g}\left(X_{j}, X_{j}\right)=1 ; \quad i=2,3,5,8 ; \quad j=1,4,6,7 ; \\
& \bar{g}\left(X_{i}, X_{j}\right)=0 ; \quad i \neq j ; \quad i, j=1,2, \ldots, 8 .
\end{aligned}
$$


It is easy to check that the so defined metric $\bar{g}$ is a Norden metric on $g l(2 ; \mathbb{C})$. Thus $(G L(2 ; \mathbb{C}), \bar{J}, \bar{g}, \overline{\widetilde{g}})$ is an 8-dimensional almost complex manifold with Norden metric. Since the metric $\bar{g}$ is left invariant, for the Levi-Civita connection $\bar{\nabla}$ of $\bar{g}$ we have

$$
2 \bar{g}\left(\bar{\nabla}_{\bar{X}} \bar{Y}, \bar{Z}\right)=\bar{g}([\bar{X}, \bar{Y}], \bar{Z})+\bar{g}([\bar{Z}, \bar{X}], \bar{Y})+\bar{g}([\bar{Z}, \bar{Y}], \bar{X})
$$

for any $\bar{X}, \bar{Y}, \bar{Z} \in g l(2 ; \mathbb{C})$. Using (62) and $\bar{J}$ is bi-invariant we get $F(\bar{X}, \bar{Y}, \bar{Z})=0$. Consequently, $(G L(2 ; \mathbb{C}), \bar{J}, \bar{g}, \overline{\widetilde{g}})$ is a Kaehler manifold with Norden metric.

Example 5.2. The unitary group $U(2)=\left\{A \in G L(2 ; \mathbb{C}): A^{-1}=\bar{A}^{T}\right\}$ is a Lie group which is a Lie subgroup of $G L(2 ; \mathbb{C})$. Hence $U(2)$ is a submanifold of $G L(2 ; \mathbb{C})$. The real Lie algebra $u(2)$ which consists of all $(2 \times 2)$ skew Hermitian matrices, i.e. $u(2)=\left\{A \in g l(2 ; \mathbb{C}): \bar{A}+A^{T}=0\right\}$ is the Lie algebra of $U(2)$ and it is a 4 -dimensional subalgebra of $g l(2 ; \mathbb{C})$. We have $u(2)=\operatorname{span}\left\{F_{1}=X_{2}, F_{2}=X_{3}-X_{5}, F_{3}=X_{4}+X_{6}, F_{4}=X_{8}\right\}$. Denote by $g$ and $\widetilde{g}$ the induced metrics on $u(2)$ of $\bar{g}$ and $\overline{\widetilde{g}}$, respectively. Using (61) we calculate $g\left(F_{1}, F_{1}\right)=g\left(F_{4}, F_{4}\right)=1 ; \quad g\left(F_{2}, F_{2}\right)=-g\left(F_{3}, F_{3}\right)=2$; $g\left(F_{i}, F_{j}\right)=0, i \neq j, i, j=1,2,3,4$. Therefore $(U(2), g)$ is a 4-dimensional non-degenerate submanifold of $G L(2 ; \mathbb{C})$. Moreover $g$ is a Lorentz metric. We find that the normal space $u(2)^{\perp}$ is spanned by $\left\{\bar{J} F_{1}, \bar{J} F_{2}, \bar{J} F_{3}, \bar{J} F_{4}\right\}$ which means that $(U(2), g)$ is a Lagrangian submanifold of $G L(2 ; \mathbb{C})$. From Theorem 3.3 it follows that $(U(2), \widetilde{g})$ is a totally lightlike submanifold of $G L(2 ; \mathbb{C})$ such that $\bar{J}(\operatorname{Rad} u(2))=\bar{J} u(2)=\operatorname{ltr}(u(2))$.

Example 5.3. The special unitary group $S U(2)=U(2) \bigcap S L(2 ; \mathbb{C})=$ $\left\{A \in G L(2 ; \mathbb{C}): A^{-1}=\bar{A}^{T}, \operatorname{det}(A)=1\right\}$ is a Lie group which is a Lie subgroup of $G L(2 ; \mathbb{C})$. Hence $S U(2)$ is a submanifold of $G L(2 ; \mathbb{C})$. The real Lie algebra $s u(2)$ which consists of all $(2 \times 2)$ traceless skew Hermitian matrices, i.e. $s u(2)=\left\{A \in g l(2 ; \mathbb{C}): \bar{A}+A^{T}=0, \operatorname{tr} A=0\right\}$ is the Lie algebra of $S U(2)$ and it is a 3 -dimensional subalgebra of $g l(2 ; \mathbb{C})$. We have $s u(2)=$ $\operatorname{span}\left\{\xi_{1}=X_{2}-X_{8}, \xi_{2}=X_{3}-X_{5}, \xi_{3}=X_{4}+X_{6}\right\}$. Using (61) we calculate $g\left(\xi_{1}, \xi_{1}\right)=g\left(\xi_{2}, \xi_{2}\right)=-g\left(\xi_{3}, \xi_{3}\right)=2, \quad g\left(\xi_{i}, \xi_{j}\right)=0, i \neq j, i, j=1,2,3$. Therefore $(S U(2), g)$ is a 3 -dimensional non-degenerate submanifold of $G L(2 ; \mathbb{C})$. Moreover $g$ is a Lorentz metric. We find that the normal space $s u(2)^{\perp}$ is spanned by $\left\{\bar{J} \xi_{2}, \bar{J} \xi_{3}, X_{1}, X_{7}, X_{2}+X_{8}\right\}$. As $\bar{J} \xi_{1}=-X_{1}+X_{7}$ belongs to $s u(2)^{\perp}$ it follows that $\bar{J} s u(2) \subset s u(2)^{\perp}$. Hence $(S U(2), g)$ is a totally real submanifold of $G L(2 ; \mathbb{C})$. The left invariant vector fields $\xi_{4}=X_{1}+X_{7}$ and $\bar{J} \xi_{4}=X_{2}+X_{8}$ belong to $s u(2)^{\perp}$. By direct calculations we check that $\bar{g}\left(\bar{J} \xi_{i}, \bar{J} \xi_{j}\right)=\bar{g}\left(\bar{J} \xi_{i}, \xi_{4}\right)=0, i \neq j, i, j=1,2,3,4$. Thus 
$\left\{\bar{J} \xi_{1}, \bar{J} \xi_{2}, \bar{J} \xi_{3}, \xi_{4}, \bar{J} \xi_{4}\right\}$ is an orthogonal basis of $s u(2)^{\perp}$ with respect to $\bar{g}$. It is clear that the complementary orthogonal vector subspace $(\bar{J} s u(2))^{\perp}$ of $\bar{J} s u(2)$ in $s u(2)^{\perp}$ is spanned by $\left\{\xi_{4}, \bar{J} \xi_{4}\right\}$ and consequently it is holomorphic. Now, from Theorem 3.3 it follows that $(S U(2), \widetilde{g})$ is an isotropic submanifold of $G L(2 ; \mathbb{C})$ such that $\bar{J}(\operatorname{Rad} s u(2))=\bar{J} s u(2)=\operatorname{ltr}(s u(2))$ and $S\left(s u(2)^{\widetilde{\perp}}\right)=(\bar{J} s u(2))^{\perp}$.

\section{References}

1. K. L. Duggal and A. Bejancu, Lightlike Submanifolds of Semi-Riemannian Manifolds and Applications, Kluwer Academic, 364, 1996.

2. K. L. Duggal and B. Sahin, Differential Geometry of Lightlike Submanifolds, Birkhäuser Verlag AG, 2010.

3. G. Ganchev and A. Borisov, Note on almost complex manifolds with Norden metric, C. R. Acad. Bulg. Sci. 39 (1986), pp 31-34.

4. G. Ganchev, K. Gribachev and V. Mihova, B-connections and their conformal invariants on conformally Kaehler manifolds with B-metric, Publications De L'Institut Mathematique, Nouvelle serie, tome 42 (56), 1987, pp 107-121.

5. G. Ganchev, K. Gribachev, V. Mihova, Holomorphic hypersurfaces of Kaehler manifolds with Norden metric. Plovdiv Univ. Sci. Works - Math., vol. 23, no. 2, (1985), pp 221236. (in Bulgarian)

6. M. Manev. Classes of real time-like hypersurfaces of a Kaehler manifold with B-metric. J. Geom., vol. 75, no. 1-2 (2002), pp 113-122.

7. M. Manev, Classes of real isotropic hypersurfaces of a Kaehler manifold with B-metric. Compt. rend. Acad. bulg. Sci., vol. 55, no. 4 (2002), pp 27-32.

8. B. Sahin, Transversal lightlike submanifolds of indefinite Kaehler manifolds, Analele Universitatii de Vest, Timisoara, Seria Matematica - Informatica, XLIV, 1, (2006), pp 119-145.

9. K. Yano and M. Kon, CR-Submanifolds of Kaehlerian and Sasakian Manifolds, Birkhäuser, 1983. 